\title{
Variegated VC Rime Restrictions in Sinitic Languages
}

\author{
Chiachih Lo, Feng-fan Hsieh, and Yueh-chin Chang \\ National Tsing Hua University
}

\section{Introduction}

Restrictions on the nucleus+coda combinations in Sinitic languages have been conventionally analyzed as co-occurrence markedness constraints, e.g., RIME HARMONY (Duanmu 2000, 2003, Lin 1989, among others) for Standard Chinese, or, *IK (*[-cons, +hi][+cons, +hi]) for Cantonese (Kenstowicz 2012, see also, e.g., Cheng 1991). However, these monolithic markedness constraints not only fail to provide a motive force behind these restrictions, but also overgenerate by predicting unattested gaps. ${ }^{1}$ To name a few, RIME HARMONY disallows the nucleus and post-nuclear elements to differ in [round] and [back] values. But violations of this (configurational) constraint are not uncommon in Sinitic languages. For example, we see in Table 1 that -ot is legitimate in Hakka and Cantonese, and - $u t$ is attested in Taiwanese Southern Min (henceforth Taiwanese). Second, no restrictions are found when the nucleus vowel is /a/ and when the coda is "placeless" (i.e., -?). Third, no account is provided for why /e/ is more restricted than /i/ in VC rimes, given that they are both front vowels. Finally, and more importantly, we can see in Table 1 that the back vowels $\{\mathrm{u}, \mathrm{o} / \mathrm{o}\}$ are in complementary distribution in Taiwanese $\mathrm{VC}$ rimes but that is not the case for the comparable VC combinations in Hakka and Cantonese. The above observations raise the question whether non-existing, impermissible VC rimes can merely be explained away by re-ranking markedness and faithfulness constraints for the rime gaps in different Sinitic languages. It is unlikely that the fact that the restrictions are more relaxed in Hakka and Cantonese is attributable to constraint re-ranking alone. Equally remarkably, it is also left unexplained in a markedness-based account as to why co-occurrence restrictions are absent only when the nucleus is /a/. Underspecification is of no avail here because the low vowel cannot be the "most unmarked" vowel. Consider now Table 1. Note that transcriptions are phonemic.

Table 1. Existing $(\checkmark)$ and non-existing $(X)$ VC combinations in Taiwanese, Hakka, and Cantonese

\begin{tabular}{|c|c|c|c|c|c|c|c|c|c|c|c|c|}
\hline Taiwanese & $-p$ & $-\overline{\mathrm{t}}$ & $-\vec{k}$ & -? & Hakka & $-\vec{p}$ & $-\vec{t}$ & $-\vec{k}$ & Cantonese & $-\vec{p}$ & $-\vec{t}$ & $-\vec{k}$ \\
\hline $\mathrm{a}$ & $\checkmark$ & $\checkmark$ & $\checkmark$ & $\checkmark$ & $\mathrm{a}$ & $\checkmark$ & $\checkmark$ & $\checkmark$ & $\{a, b\}$ & $\checkmark$ & $\checkmark$ & $\checkmark$ \\
\hline $\mathrm{i}$ & $\checkmark$ & $\checkmark$ & $\checkmark$ & $\checkmark$ & i & $\checkmark$ & $\checkmark$ & $x$ & $\mathrm{i}$ & $\checkmark$ & $\checkmark$ & $\checkmark$ \\
\hline e & $x$ & $\checkmark$ & $x$ & $\checkmark$ & e & $\checkmark$ & $\checkmark$ & $x$ & $\mathrm{e}$ & $\checkmark$ & $x$ & $\checkmark$ \\
\hline $\mathrm{u}$ & $x$ & $\checkmark$ & $x$ & $\checkmark$ & $\mathrm{u}$ & $x$ & $\checkmark$ & $\checkmark$ & $\mathrm{u}$ & $x$ & $\checkmark$ & $\checkmark$ \\
\hline$\{0,0\}$ & $\checkmark$ & $x$ & $\checkmark$ & $\checkmark$ & o & $x$ & $\checkmark$ & $\checkmark$ & o & $x$ & $\checkmark$ & $\checkmark$ \\
\hline
\end{tabular}

\footnotetext{
* This study was supported by National Science Council projects 100-2628-H-007-036-MY2 and 97-2410-H-007-025-, to which we are grateful.

1 These rime gaps may be either an accidental or a systematic gap. See Section 5.4 for more discussion.
} 


\section{Working Hypothesis}

Since stop codas are never released in Chinese languages, the most salient cue for place is absent. Following Hsieh (2010), we assume that, unlike many other languages, Chinese VC rimes involve a larger degree of coarticulation ${ }^{2}$ so that the nucleus vowel may serve as an "indicator" for the place of articulation of a coda by means of (contrast-enhancing) vowel quality change. Meanwhile, vowel reduction (as a result of target undershoot) occurs because vowel duration is significantly diminished in VC rimes (Flemming 2004). Taken together, at least in Taiwanese, vowel reduction, or, centralization, endangers vowel contrasts, hence the gaps (i.e., shaded cells with a $X$ in Table 1). To this end, a duration-based analysis predicts that there should be a significant difference between Taiwanese and Hakka/Cantonese in the acoustic properties of VC rimes, but not in the (dis-)preferred co-occurrence constraints. Therefore, one of the goals of the present study is to investigate if this prediction can be experimentally confirmed.

\section{Experiments}

\subsection{Speakers and Material}

3.1.1 Taiwanese Ten male native Taiwanese speakers participate in the experiment. Five speakers were born between 1976 and 1980 and represent the younger generation. Five were born before 1975 (among whom three were born before 1960), representing an older generation. Five of them are from southern Taiwan, and the other five are from northern Taiwan.

The test material includes all 30 rimes in Taiwanese and is divided into three syllable types: CV, CVN, and CVS(top). The nuclei include $\{\mathrm{i}, \mathrm{e}, \mathrm{a}, \mathrm{u}, \mathrm{o}, \mathrm{\rho}\}$ in open syllables and $\{\mathrm{i}, \mathrm{e}, \mathrm{a}, \mathrm{u}, \mathrm{\rho}\}$ in closed syllables. Three nasal codas $\{\mathrm{m}, \mathrm{n}, \mathrm{p}\}$, and four stop codas $\{\mathrm{p}, \mathrm{t}, \mathrm{k}, \mathrm{P}\}$ are used. The onsets consist of the three basic voiceless unaspirated stops $\{\mathrm{p}, \mathrm{t}, \mathrm{k}\}$. Sibilants and glottal fricative $\{\mathrm{ts}, \mathrm{s}, \mathrm{h}\}$ are also used when available. In this experiment, $\mathrm{CV}$ and $\mathrm{CVN}$ syllables are combined with the high level tone. If such a combination is not available, mid-level tone and falling tone are used instead. CVS syllables are pronounced with both high and low checked tones. 12 tokens (with different onsets and tones) are prepared for each rime and are repeated three times. Due to the differences of age and/or region, some speakers do not have certain rimes such as /o?/, /e $/$ /, /o?/, /op/ and/or /om/. Therefore, the total number of tokens for each participant ranges from 1,032 to 1,080. All target words are monosyllables and are embedded in the carrier sentence "t6it li 6i _ " (this word is __).

3.1.2 Hoiliuk (Hailu) Hakka Five male native speakers of Hoiliuk (Hailu) Hakka are recruited in this study. All speakers were over 50 years old at the time of recording. Target words are all monosyllables comprising the 27 possible rimes of Hakka. All 7 tones in the language are chosen, with high register tones prioritized. Each rime contains an average of 14 target words (depending on availability of meaningful words). With three repetitions, each speaker produced 1,098 tokens. The target words are embedded in the carrier phrase "lia tfak sı he _." (this word is __).

3.2 Recording and Data Analysis The recordings were conducted in the sound-proof room at the phonetics lab of the Institute of Linguistics, National Tsing Hua University, using a Shure Beta head worn microphone connected to a Roland R09HR Recorder. The target words appear randomly on a computer screen. The participants firstly spent some time to be familiarized with the target words because the stimuli normally may not appear in written forms. Then the speakers were instructed to proceed at a normal speech rate for the recording.

The acoustic data were analyzed with the help of Praat (Boersma and Weenink, 2010). Each token was divided into 6 equal portions (or, 7 points). Formant values were measured from point 4 (midpoint) to point 7 (endpoint). The obtained formant values were subsequently normalized by means of Lobanov method, using a web-based software NORM (Thomas and Kendall, 2007) and were converted back to Hz.

\footnotetext{
2 Chen et al.'s (2013) EMA results show a substantially larger degree of coarticulation between the vowel and the stop coda in Taiwanese, if compared with their counterparts in non-Sinitic languages, i.e., English and Persian, reported in Modarresi et al. (2004).
} 


\section{Results}

\subsection{Vowel Duration}

4.1.1 Mean Duration \& CVS/CV Ratios The results show that Taiwanese CVS syllables are significantly shorter than the comparable short syllables in Hakka and the half-long syllables (CV:S) in Cantonese (all Cantonese data in this study are adapted from Zee 2003). The CVS /CV (or CV:S/CV) ratios are $42.9 \%, 54.88 \%$, and $57.14 \%$ for Taiwanese, Hakka, and Cantonese, respectively. The difference in $\mathrm{CVS} / \mathrm{CV}$ ratio between Taiwanese and Hakka is statistically significant $(p<0.001)$.

Table 2. Mean duration of three syllable types and CVS/CV ratios in Taiwanese and Hakka (in milliseconds)

\begin{tabular}{|l|l|l|l|l|l|l|}
\hline & \multicolumn{2}{|l}{ laiwanese } & Hakka & \multicolumn{3}{l|}{ Cantonese } \\
\hline & mean duration & SD & mean duration & SD & \multicolumn{2}{l|}{ mean duration } \\
\hline CV & 217.72 & 43.8 & 226.63 & 57.8 & CV & 350 \\
\hline CVN & 132.19 & 30.2 & 159.51 & 49.0 & CV:S & 200 \\
\hline CVS & 93.409 & 21.8 & 124.38 & 46.4 & CVS & 120 \\
\hline CVS/CV (\%) & $42.9 \%$ & & $54.9 \%$ & & CV:S/CV (\%) & $57.1 \%$ \\
\hline
\end{tabular}

4.1.2 Durational Properties Looking closer into the data of individual rimes in Table 3, we found that the durational patterns of Taiwanese closed syllables also differ from those of Hakka. In Taiwanese, $\{-\mathrm{ik}$, -ut $\}$, group together as the longest rimes, followed by $\{$-et, -at\} and $\{-\mathrm{ak},-\mathrm{it},-\mathrm{ap},-\mathrm{ok}\}$, while $\{-\mathrm{ip}\}$ is the shortest. The distribution suggests that a combination of $\mathrm{VC}$ with opposite values in [back] (e.g.,/-ik/and /-ut/) requires more time to produce. Second, /-at/ and /-ap/ are significantly longer than /-it/ and /-ip/, respectively, indicating that low vowels are longer than high vowels when combined with the same coda. Lastly, rimes closed by a labial coda are shorter than those by coronal and velar codas. So we can say that rime duration in Taiwanese correlates closely with degree of coarticulation. That is perhaps why Vp stands out as the shortest - it is well-established that labial consonants, compared with coronal and velar ones, have a lower degree of coarticulation with neighboring vowels (See, e.g., Linblom 1963, among many others).

Table 3a. Rime duration of Taiwanese (durational differences among the rimes marked with the same horizontal bars are statistically insignificant $(p>0.05))$

\begin{tabular}{|c|c|c|c|c|c|c|c|c|c|}
\hline Rime & lik & ut & et & at & ak & it & ap & ok & ip \\
\hline Duration (ms) & 107.5 & 102.4 & 99.4 & 99.3 & 92.8 & 91.5 & 91.4 & 90.2 & 79.2 \\
\hline SD & 23.7 & 22.2 & 16.8 & 17.6 & 22.9 & 18.3 & 20.9 & 18.9 & 19.0 \\
\hline Statistical & & & & & & & & & \\
\hline significance & & & & & & & & & \\
\hline
\end{tabular}


Table 3b. Rime duration of Hakka (durational differences among the rimes marked with the same horizontal bars are statistically insignificant $(p>0.05))$

\begin{tabular}{|c|c|c|c|c|c|c|c|c|c|c|c|}
\hline Rime & ot & ok & et & it & ap & ip & ut & at & ep & uk & ak \\
\hline Duration (ms) & 138.0 & 132.6 & 129.7 & 126.6 & 124.5 & 124.5 & 121.7 & 120.8 & 119.2 & 118.3 & 112.2 \\
\hline SD & 58.5 & 52.3 & 52.5 & 49.3 & 45.8 & 51.4 & 61.3 & 49.8 & 47.9 & 47.1 & 39.6 \\
\hline Statistical & & & & & & & & & & & \\
\hline significance & & & & & & & & & & & \\
\hline
\end{tabular}

On the other hand, the durational differences among most Hakka rimes are, grosso modo, not significant. Neither could we find a positive correlation between rime duration and degree of VC coarticulation. In contrast to Taiwanese, $\{-\mathrm{ot},-\mathrm{ok}\}$ in Hakka stand out as the longest rimes in the inventory. This is probably due to a strategy adopted by Hakka speakers to enhance vowel perception, to which we shall return in Section 6.

\subsection{Formants and Gliding}

4.2.1 Formant Transitions In Chinese languages, F2 transitions in VC rimes are crucial for distinguishing among places of articulation (recall that stop codas are never released). Our experimental results show that the formant transitions are different in the magnitude between Taiwanese and Hakka closed syllables, as in Figures 1a and 1b.

\section{Taiwanese}

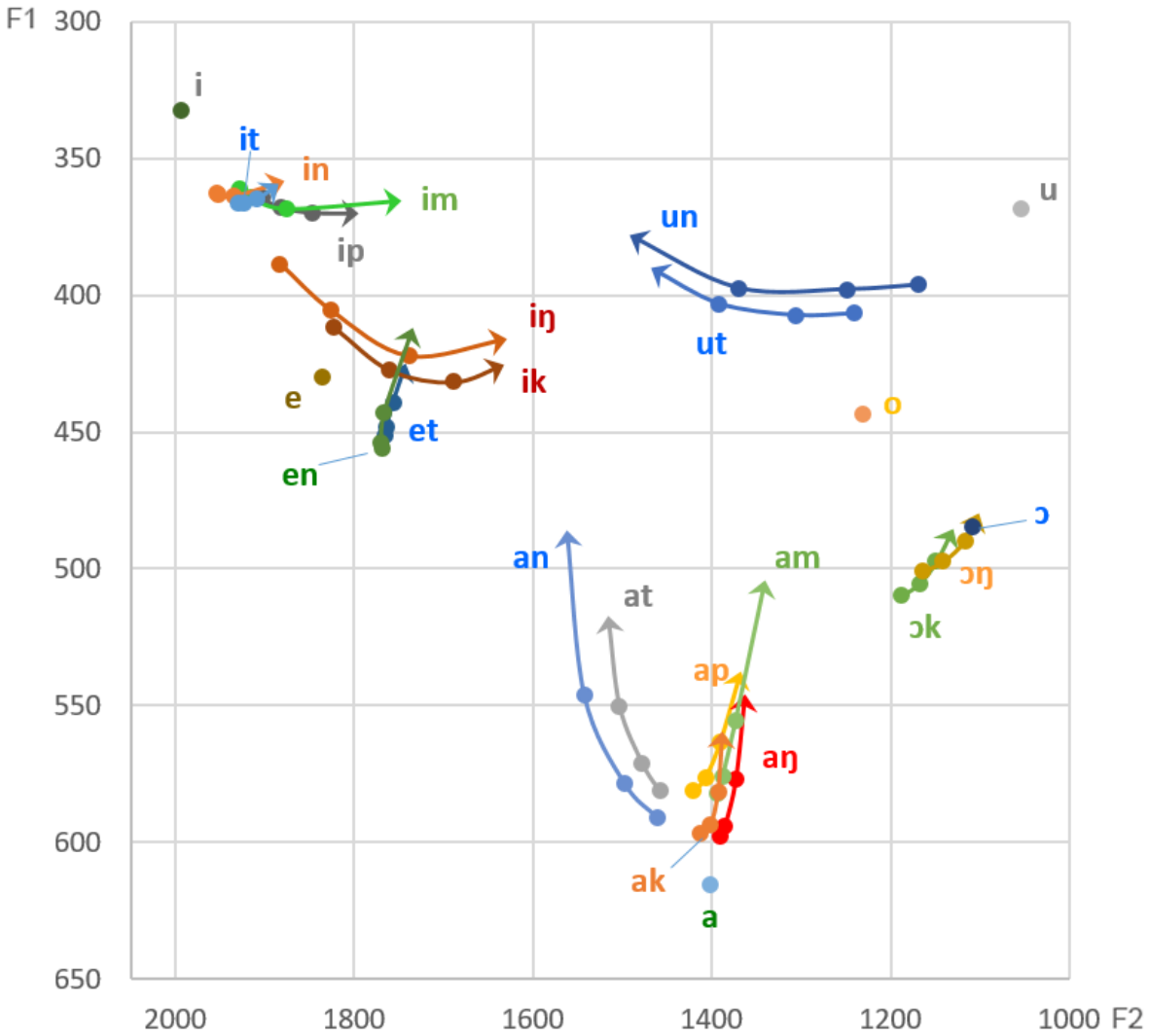


Figure 1a. Formant trajectories of Taiwanese on the F1/F2 vowel plane. Trajectories are drawn from the midpoint to the endpoint (indicated by an arrowhead) of vowels. Each interval segmented by two adjacent points is equal in temporal duration. The formant values of each point are Lobanov-normalized and converted back to Hz. Transcriptions are phonemic.

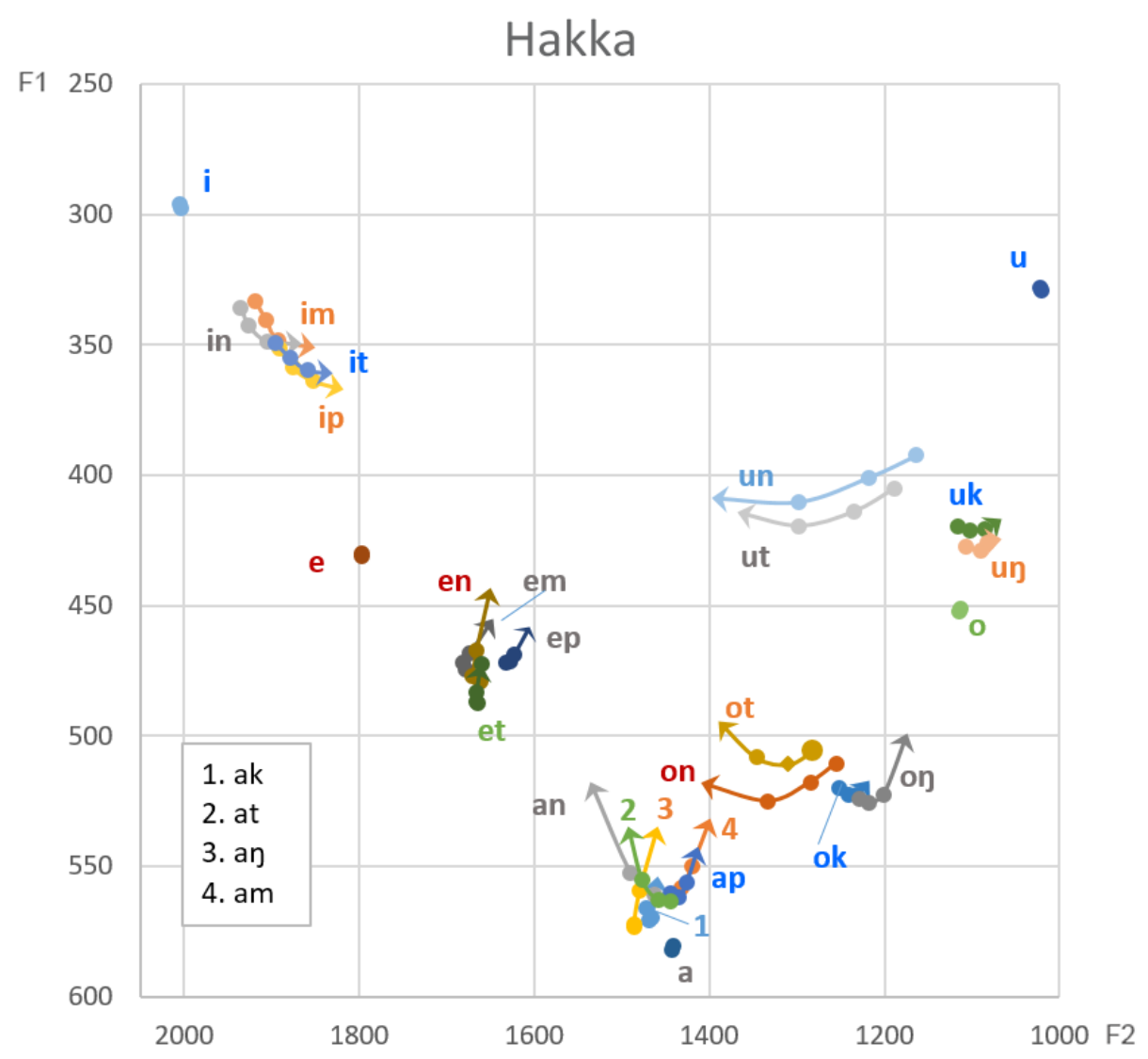

Figure 1b. Formant trajectories of Hakka on the F1/F2 vowel plane.

Some generalization can be obtained from both the Taiwanese and Hakka data. First, the trajectories of each VN/VS pair run parallel to each other, with the trajectory of VS more centralized than that of the corresponding VN. Second, high vowels $/ \mathrm{i} /$ and $/ \mathrm{u} /$ move toward the center when followed by $/ \mathrm{k} /$ and $/ \mathrm{t} /$ respectively, and low vowel /a/ primarily undergoes raising in closed syllables. Third, the F2 transitions of /-ik/ and /-ut/ are significant, leading to the emergence of a diphthongized (short) vowel in these contexts. Moreover, mid vowels $\{\mathrm{e}, \mathrm{o}\}$ exhibit less pronounced formant movement than high vowels (see (4b) below for details). Lastly, the magnitude of formant movement is significantly greater in Taiwanese than in Hakka (see (4a) below for details). The length of transition is the linear distance on the F1/F2 vowel plane from the vowel midpoint to offset. F2 variations in (4b) are calculated by F2 at vowel offset minus F2 at vowel midpoint (as a result, a negative number represents a vowel moving to a fronted position, and a positive number indicates a vowel moving to a back position). 
Table 4. Length of transition and F2 variations in Taiwanese and Hakka checked syllables

Length of transitions (in $\mathrm{Hz}$ )

\begin{tabular}{|c|c|c|}
\hline Rime & Taiwanese (SD) & Hakka (SD) \\
\hline it & $58(28)$ & $66(31)$ \\
\hline ip & $118(56)$ & $75(48)$ \\
\hline ik & $238(52)$ & N.A. \\
\hline et & $53(27)$ & $19(8)$ \\
\hline ep & N.A. & $27(11)$ \\
\hline at & $98(40)$ & $60(28)$ \\
\hline ap & $88(41)$ & $35(12)$ \\
\hline ak & $60(46)$ & $23(15)$ \\
\hline ot & N.A. & $110(65)$ \\
\hline ok & $66(21)$ & $35(16)$ \\
\hline ut & $229(69)$ & $163(67)$ \\
\hline uk & N.A. & $50(14)$ \\
\hline Average & $110(79)$ & $60(53)$ \\
\hline
\end{tabular}

F2 variations (in $\mathrm{Hz}$ )

\begin{tabular}{|c|c|c|}
\hline Rime & Taiwanese (SD) & Hakka (SD) \\
\hline it & $-51(37)$ & $-65(31)$ \\
\hline ip & $-117(56)$ & $-73(48)$ \\
\hline ik & $-236(51)$ & N.A. \\
\hline et & $-29(28)$ & $-8(4)$ \\
\hline ep & N.A. & $-21(12)$ \\
\hline at & $55(45)$ & $50(25)$ \\
\hline ap & $-62(37)$ & $-30(10)$ \\
\hline ak & $-19(20)$ & $-14(10)$ \\
\hline ot & N.A. & $109(64)$ \\
\hline ok & $-56(21)$ & $-34(16)$ \\
\hline ut & $229(70)$ & $163(67)$ \\
\hline uk & N.A. & $-49(14)$ \\
\hline Average & $95(40)$ & $56(27)$ \\
\hline
\end{tabular}

We can also see from (4) that the labial coda suppresses F2 across the board. The velar coda is more compatible with the low and back vowels, and less compatible with the front vowels. The coronal coda is generally compatible with front vowels, but less so with the low/back vowels. Compatibility of VC sequences correlates with the change in F2 because more compatible $\mathrm{VC}$ rimes involve less movement of the tongue body and induce only slight changes in F2 values.

4.3 Vowel Laxing in Closed Syllables Recall from Table 2 that vowel duration decreases in an order in these syllable structures: $\mathrm{CV}>\mathrm{CVN}>\mathrm{CVS}$. The vowel space (measured from the midpoint of nucleus vowels), too, shrinks in the same order. The differences are statistically significant $(p<0.001)$ in terms of (i) vowel space area and (ii) Formant Centralization Ratio (FCR) in both Taiwanese and Hakka (See Figures 2).

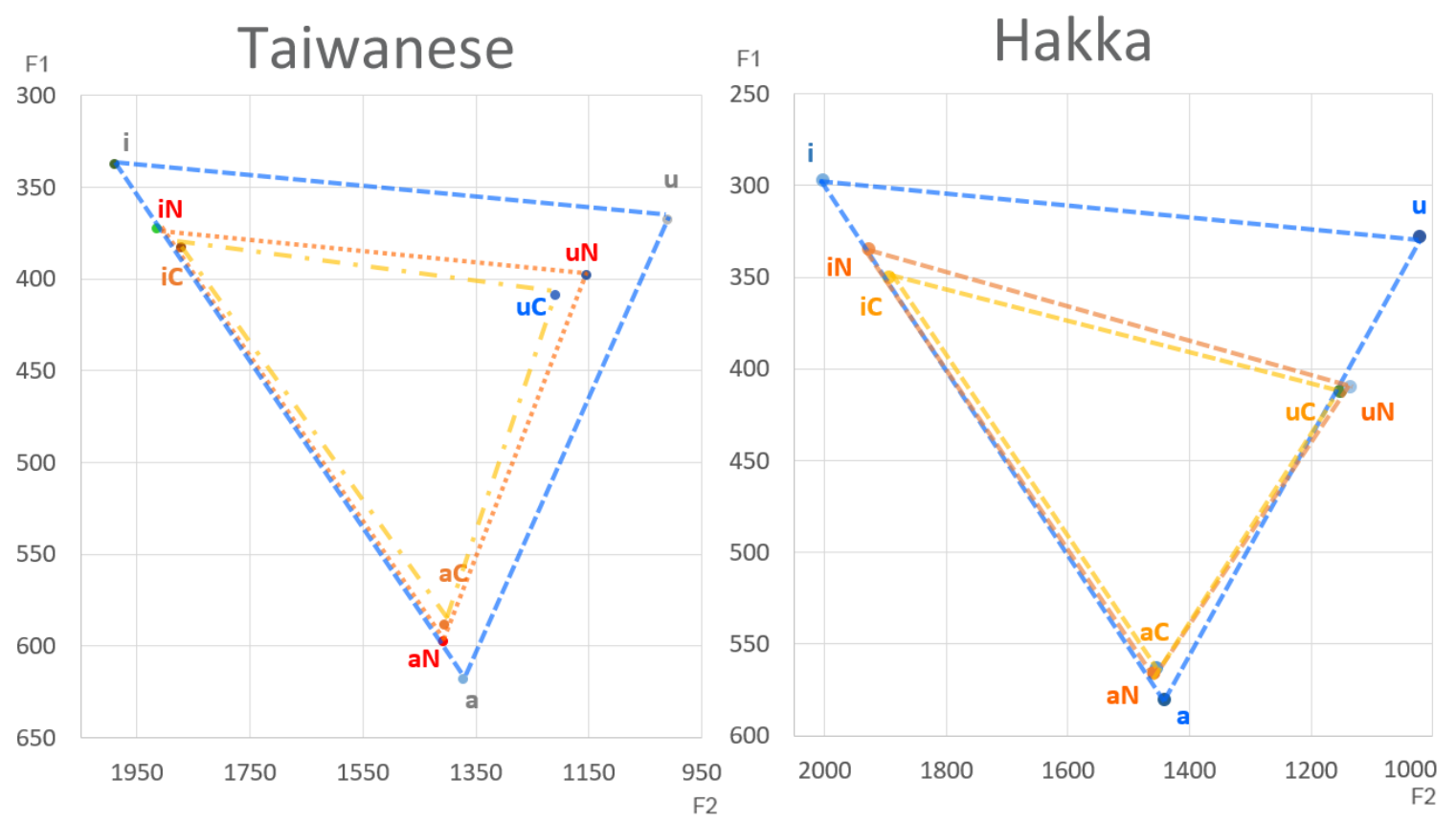

Figure 2. Vowel spaces of CV, CVN \& CVS in Taiwanese and Hakka. 
In addition, an asymmetry is observed in terms of the degree of laxing in the front and back vowels in Hakka. Figure 3 indicates that the vowel space of Hakka closed syllables collapses more radically on the side of back vowels. A detailed examination of the laxing degree by means of F1 change is provided in Table 5 and in Figure 4. As we can see, the velar coda "drags" the vowel downward more than coronal and labial codas do. The laxing degree in Table 5 is obtained by F1 at vowel midpoint in CVS/CVN syllables minus F1 at vowel midpoint in the corresponding CV syllables.

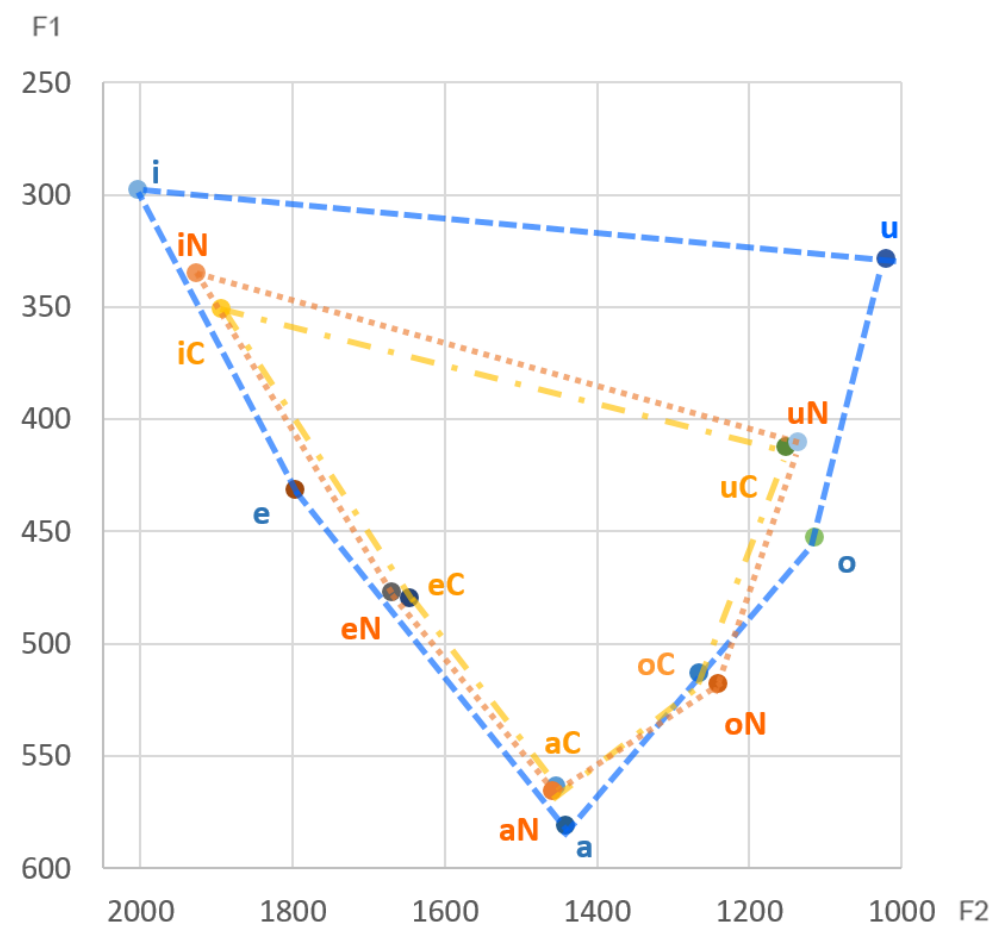

Figure 3. Vowel space of Hakka (non-corner vowels included).

Table 5. Laxing degrees of Taiwanese and Hakka closed syllables (in $\mathrm{Hz}$ )

\begin{tabular}{|c|c|c|c|c|c|}
\hline VC & Taiwanese (SD) & Hakka (SD) & VN & Taiwanese (SD) & Hakka (SD) \\
\hline it & $29(16)$ & $51(7)$ & in & $26(10)$ & $38(11)$ \\
\hline ip & $29(11)$ & $54(11)$ & im & $26(10)$ & $36(10)$ \\
\hline ik & $79(23)$ & N.A. & in & $51(25)$ & N.A. \\
\hline et & $24(14)$ & $54(11)$ & en & $27(15)$ & $45(19)$ \\
\hline ep & N.A. & $41(11)$ & em & N.A. & $45(22)$ \\
\hline at & $-35(17)$ & $-20(13)$ & an & $-25(13)$ & $-19(13)$ \\
\hline ap & $-37(14)$ & $-26(17)$ & am & $-31(14)$ & $-20(11)$ \\
\hline ak & $\mathbf{- 2 3 ( 1 7 )}$ & $\mathbf{- 1 5}(22)$ & an & $-18(14)$ & $-11(11)$ \\
\hline ot & N.A. & $54(12)$ & on & N.A. & $58(15)$ \\
\hline ok & $28(14)$ & $\mathbf{6 8}(14)$ & on & $20(17)$ & $\mathbf{7 2}(13)$ \\
\hline ut & $38(13)$ & $75(16)$ & un & $29(9)$ & $65(23)$ \\
\hline uk & N.A. & $\mathbf{9 2 ( 2 1 )}$ & un & N.A. & $\mathbf{1 0 0}(23)$ \\
\hline
\end{tabular}




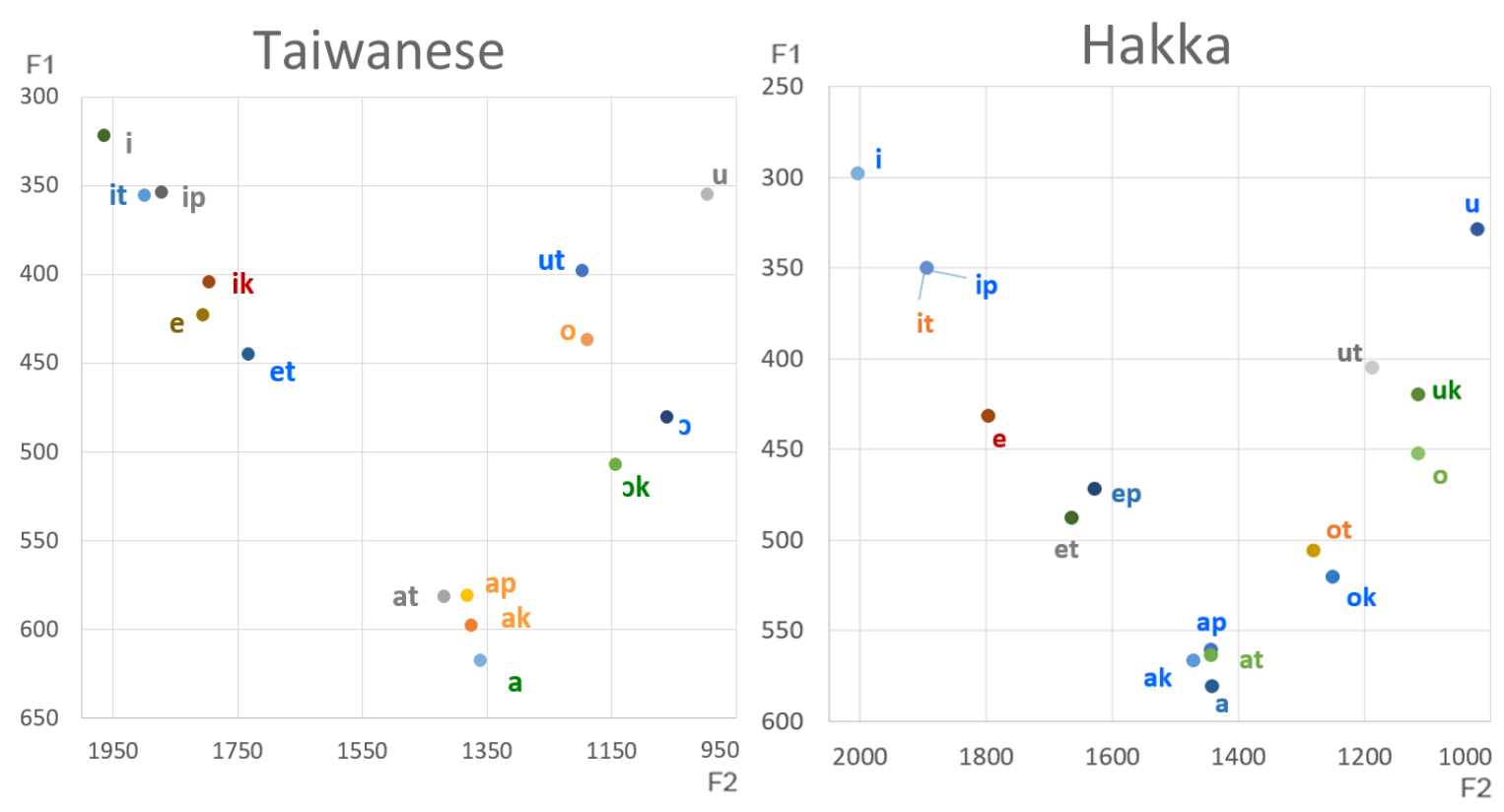

Figure 4. Vowels (measured from the midpoint) in CV \& CVC syllables of Taiwanese and Hakka on the F1/F2 vowel plane. Formant values are Lobanov-normalized and converted back to Hz. Note that the midpoints of /ip/ and /it/ in Hakka overlap completely.

\section{Discussion}

5.1 Reconsidering the Role of Duration Hsieh (2010) proposes a duration-based account for rime gaps in Sinitic languages. The gist is that since stop codas are invariably unreleased, perceptibility of places of articulation is endangered. Hsieh (2010) further argues that closed syllable laxing, caused by reduced vowel duration, serves as the other motive force behind the restrictions on VC rimes. When distinctiveness is difficult to maintain in a diminished vowel space, vowel contrasts are subject to merger (Flemming 2004).

In this study, however, we found some unexpected results. First, both Taiwanese and Hakka are found to exhibit pronounced vowel laxing in closed syllables (Figures 2a, b). Vowel laxing alone does not seem to suffice in explaining the disparities of VC phonotactics in the two languages (recall from Table 1 that /-ot/ and /-uk/ are attested in Hakka, but not in Taiwanese). Furthermore, a closer examination reveals a tantalizing fact that diphthongization (or vowel gliding), presumably more effortful in articulation, is more pronounced in Taiwanese VC rimes. By contrast, vowel gliding is surprisingly less pronounced in Hakka VC rimes, which are in fact longer in duration (4a). In other words, these observations seem to vitiate the role of duration in vowel reduction.

5.2 Perceptual difficulties in Chinese closed syllables Due to robust vowel reduction found in Chinese closed syllables, vowel distinctiveness may be weakened. In response, Hakka opts for lengthening the duration of perceptually less salient vowel to maintain contrast, whereas Cantonese uses the vowel length contrast to distinguish between potentially confusable vowels. Both strategies are nonetheless not available to Taiwanese due to the exceptionally short duration of $\mathrm{VC}$ rimes, which, we believe, gives rise to the gaps in the $\mathrm{VC}$ rimes. Meanwhile, the extra-short duration of $\mathrm{VC}$ rimes also reduces the F2 transitions and endangers perceptibility of places of articulation of coda consonants. So we posit that this unique phonetic property of Taiwanese $\mathrm{VC}$ rimes (i.e., cross-linguistically shorter phonetic length) has a bearing on gliding (or, diphthongization; see Figure 1a) and vowel mergers, to which we return in the following sub-sections.

5.2.1 Problem 1: Perceptibility of Places of Articulation of Codas Recall from Table 2 that Taiwanese CVS syllables are not just shorter than CV syllables, but are significantly shorter than the comparable CVS syllables of Hakka and Cantonese in both duration and CVS/CV ratio. As duration decreases, $\mathrm{VC}$ transitions are harder to maintain, making codas harder to perceive. In response, Taiwanese 
speakers opt for stronger VC coarticulations to maintain place contrasts for codas. Such effort can also be observed in the lengthened duration of syllables with "incompatible" VC sequences, namely, syllables containing /-ik/ and /-ut/ (see Table 3 above). In contrast, no such effect is attested in Hakka.

5.2.2 Problem 2: Vowel Distinctiveness Vowel contrasts may become more confusable as vowel space shrinks. However, Hakka still preserves a full contrast between $/ \mathrm{u} / \mathrm{and} / \mathrm{o} /$ in closed syllables. Under scrutiny, it reveals that $\{$-ok, -ot $\}$ in Hakka are pushed substantially downwards in order to remain sufficiently dispersed with respect to the $\{$-uk, -ut $\}$ (Figures 1(b), 4(b)). So an immediate question arises: why is this option not possible for Taiwanese? The clue is hidden in the quantity and quality of the mid back vowel in in Hakka closed syllables. Located midway between -o and -a, the nucleus of \{-ok, -ot $\}$ in Hakka should be categorized as a low vowel rather than a mid vowel not only because they are low in the vowel plane, but also because the vowel trajectories of $\{$-ok, -ot $\}$ run "upwards" (more centralized) in the vowel space than those of $\{-$ oy, - on $\}$ (see Figure 1(b)), a typical reduction pattern for low vowels. According to Crosswhite's (2004) licensing-under-stress approach, increased exposure of any stimulus enhances the likelihood that the stimulus will be correctly identified. She argues that non-corner vowels, lacking the special quality of $\{\mathrm{i}$, a, $\mathrm{u}$ \}, are licensed only in the context of a stressed position where syllable duration is longer. In other words, non-corner vowels are perceptually less distinct and need longer duration to be correctly identified. By the same token, the short duration of Hakka closed syllables must be phonetically lengthened to enhance the distinctiveness of the non-corner low vowel in $\{-\mathrm{ok},-\mathrm{ot}\}$. This strategy is not possible for Taiwanese due to the much shorter duration of its checked syllables. Taken together, the extraordinarily short duration of Taiwanese closed syllables not only jeopardizes the perceptibility of places of articulation of a coda, which can be fixed by stronger $\mathrm{VC}$ coarticulation, but also precludes the possibility of maintaining vowel contrasts in a collapsed vowel space caused by closed-syllable laxing.

5.3 More on the Gaps Armed with the above, we offer an analysis of why the two gaps *-uk and *-ot are not possible in Taiwanese.

5.3.1 *-uk in Taiwanese *-uk and -ok are contextually neutralized to -ok in Taiwanese due to reduced contrast as a result of the shrunken vowel space. According to Flemming's (2004) Dispersion Theory, /o/, instead of /u/, surfaces as the optimal vowel when followed by /k/ because, theoretically, /-ok/, rather than */-uk/, is more dispersed with the "neighboring" rimes /-ut/ and /-ak/ (see Figure 4(a)). Why does this not happen to the front vowels $\{$-it, -et\}? Recall from Figures 2 and 3 that the vowel spaces of closed syllables reduce unevenly with respect to back and front vowels - a result of different laxing degrees created by different codas. Table 5 indicates that velar codas cause F1 to raise drastically for non-low vowels ${ }^{3}$. In contrast, coronal codas do not show such an effect and keep their nuclei comparatively proximate to those in the CV syllables. As a result, the vowel spaces of Taiwanese and Hakka on the side of front vowels in closed syllables are just mildly reduced, allowing $\{$-it, -et $\}$ to remain sufficiently dispersed, hence without mergers.

5.3.2 Compatibility of VC gestures As discussed in Section 4, in general, more salient $\mathrm{F} 2$ transitions, which indicate higher degrees of mutual independence of $\mathrm{VC}$ rimes, correspond to longer syllable durations in Taiwanese. This means the tongue body requires longer time to move horizontally to achieve the front and back VC targets. In contrast, more compatible VC sequences produce less pronounced F2 transitions and then are shorter in phonetic length. That is why /-ak/, /-it/, and /-ok/ are the shortest in duration in Taiwanese apart from those with a labial coda (see Table 3a). The rimes with compatible VC sequences are easier to produce and require fewer efforts. RHYME HARMONY posited by Duanmu's (2000) is a constraint that only allows the combination of a front vowel with a coronal nasal consonant, or the combination of a back vowel with a velar nasal consonant. A physiological reason has been offered to motivate this constraint: "an articulator cannot move fast enough to execute two opposite features in succession (such as [+back][-back])". This constraint is, however, violable in Taiwanese and Hakka, where a front vowel can precede a velar coda, and a back vowel, a coronal coda. Therefore, according to our analysis, RHYME HARMONY is probably

\footnotetext{
${ }^{3}$ Such an effect induced by velar codas might be attributed to the constriction site of Chinese velar codas, which, we suspect, is formed at a relatively further back position. Given that, the tongue dorsum is not required to raise too high to form a velar closure, as opposed to the constriction sites of labial and coronal stops.
} 
motivated by a different, perceptually-based reason: compatible VC sequences ([+back][+back] or [-back] [-back]) are favored not just because they are easier to produce, but also because the quality of the nucleus vowel readily indicates the identity of the following coda. Mou (2006) reported, in a gating experiment, that her subjects could identify the place of articulation of the upcoming nasal coda at the $3^{\text {rd }}$ gate in a total of 5 gates. Chu's (2009) misperception experiments also find that /t/ is the least favored coda after a low vowel in Chaoshan, a distinct group of dialects of Southern Min Chinese, and tends to be neutralized with the most favorable coda $/ \mathrm{k} /$ in the low vowel context. Hsieh et al. (2009) hold that "RHYME HARMONY is a genuine process of Mandarin grammar — an enhancement effect (Keysers and Stevens 2006) that speakers can utilize to identify the place of articulation of the nasal coda". The present study confirms that this enhancement effect also applies to the nucleus+coda combinations in other Sinitic languages, such as Taiwanese and Hakka.

5.3.3 *-ot/*-ek in Taiwanese For "incompatible" VC sequences, reliable perception of codas relies heavily on formant transitions, especially F2 transitions. The change of F2 in closed syllables in Taiwanese and Hakka shown by (4b) can be summarized in Table 6 below.

Table 6. Comparisons of F2 transition in Taiwanese and Hakka closed syllables.

\begin{tabular}{|c|c|c|c|}
\hline Coda & $\begin{array}{c}\text { Coronal } \\
{[- \text { back }]}\end{array}$ & Labial & $\begin{array}{c}\text { Velar } \\
{[+ \text { back }]}\end{array}$ \\
\hline Front [-back] & minimum & medium & maximum \\
\hline Back [+back] & maximum & medium & minimum \\
\hline High & \multicolumn{3}{|c|}{ more pronounced } \\
\hline Non-high & less pronounced \\
\hline
\end{tabular}

The F2 transition is at its greatest magnitude when the VC sequences are of opposite [back] values, and is insignificant when VC share the same [back] value. More importantly, high vowels are capable of producing greater F2 transitions than non-high vowels. Recall from our discussion in 5.2.1: Taiwanese produces stronger VC coarticulation to enhance coda perceptibility since VC transitions are harder to detect in extrashort syllables. The need for more pronounced F2 transitions in Taiwanese closed syllables precludes the VC combination of a mid vowel and a coda with opposite [back] values, such as /-ot/ and /-ek/.

5.3.4 *-ik/*-ek in Hakka Restrictions on the combination of incompatible VC rimes are asymmetric in Hakka with respect to the front vs. back vowels. While $\left\{*_{-}\right.$ik, $*_{-}$-ek $\}$are missing in the rime inventory, $\{-$ ut, -ot $\}$ are attested. A probable explanation lies in articulation: the tongue root is more 'bulky' and travels more slowly than the tongue tip. This is also indicated by the longer duration and greater F2 transition of /-ik/ than those of /-ut/ in Taiwanese (see Table 3a and (4)). In several languages, as Gick and Wilson (2006) observe, when a coda consonant with a retracted tongue root follows a front vowel, different strategies are employed to avoid a gestural conflict. One of them is to change the coda consonant into a coronal to match the advanced tongue root of a front vowel. We think this is exactly the case for Hakka, too. Specifically, the codas in $\left\{*_{-i k} *_{-i n} *_{-e k} *_{-e}\right\}$ in Hakka merge with $\{$-it, -in, -et, -en $\}$ respectively, since, diachronically speaking, these codas are velar in Middle Chinese (as well as in other Chinese languages).

5.4 The gaps: systematic or accidental? So far, the assumption we adopted in this study is that these non-existing VC rimes are ruled out by the grammatical factors. As raised by the reviewers, it may well be the case that some of the gaps are merely accidental. Furthermore, Yu (2017) points out that distribution of sound combinations does not constitute evidence of speakers' knowledge of phonotactics per se, and the absence of certain sound combinations cannot be deemed as evidence for active phonotactics constraint while some gaps may be filled readily by speakers. We believe that at least some of the gaps discussed in this work are systematic gaps even though no experimental results are available, to our knowledge. Our anecdotal observations are that Taiwanese speakers do have difficulties with $\left\{*_{-}\right.$ot, $\left.*_{\text {-on, }} *_{- \text {-uk, }} *_{- \text {uy }}\right\}$ both in perception 
and production. For example, when confronted with $/ *$-uk/, a Taiwanese speaker will produce /-ok/, not /*-uk/. This is very different from the case of English-to-Cantonese loanwords. For instance, the famous labial dissimilation is not obeyed in pem 'pump'. Consequently, it is fair to say that at least some of the gaps do require further explanations. Of course, more experimental work is needed for a deeper understanding of this issue.

\section{Conclusion}

In this study, we have argued, from a perceptual perspective, why restrictions on Chinese VC rimes should be more stringently enforced. Our data show a negative correlation between syllable duration and the number of rime gaps in Chinese languages - the longer the duration of closed syllables, the fewer rime gaps in the language. The duration-based approach provides a more satisfactory account for the motive force behind the variegated rime gaps in Sinitic languages, which cannot be simply attributed to co-occurrence markedness constraints.

\section{References}

Bauer, Robert S. 1985. The Expanding Syllabary of Hong Kong Cantonese. Cahiers de linguistique Asie orientale 14: 99-113.

Bauer, Robert S. \& Benedict, Paul K. 1997. Modern Cantonese Phonology. Berlin: Mouton De Gruyter.

Boersma, Paul and Weenink, David (2010). Praat: doing phonetics by computer [Computer program]. http://www.praat.org/

Chen, Yi-cheng, Chen, Wei-rong, Chang, Yueh-chin and Hsieh, Feng-fan. 2013. An EMA Study of Onset-Vowel vs. Vowel-Coda Coarticulation in Taiwanese Southern Min. In Lee, Wai-Sum (ed.), Proceedings of the International Conference on Phonetics of the Languages in China (ICPLC-2013), pp. 101-3. [In Chinese]

Cheng, Lisa L.-S. 1991. Feature Geometry of Vowels and Co-occurrence Restrictions in Cantonese. Proceedings of the Ninth West Coast Conference on Formal Linguistics, 107-124.

Chu, Man-ni. 2009. Motivating the Change of Stop Codas in Chaoshan: A Perceptual Study. Doctoral dissertation, National Tsing Hua University.

Crosswhite, Katherine. 2004. Vowel Reduction. Phonetically Based Phonology. Cambridge University Press pp. 191231.

Duanmu, San. 2000. The Phonology of Standard Chinese. Oxford: Oxford University Press.

Duanmu, San. 2003. The Syllable Phonology of Mandarin and Shanghai. Proceedings of the Fifteenth North American Conference on Chinese Linguistics pp. 86-102.

Duanmu, San. 2007. The Phonology of Standard Chinese. $2^{\text {nd }}$ edition. Oxford University Press.

Flemming, Edward. 2002. Auditory Representations in Phonology. New York: Garland Publishing Inc.

Flemming, Edward. 2004. Contrast and Perceptual Distinctiveness. Phonetically based phonology, 232-276.

Gick, Bryan. and Wilson, Ian. 2006. Excrescent Schwa and Vowel Laxing: Cross-Linguistic Responses to Conflicting Articulatory Targets. Laboratory phonology 8: 635-659.

Hashimoto, Oi-kan Yue. 1972. Phonology of Cantonese. Cambridge University Press.

Hsieh, Feng-fan., Kenstowicz, Michael., \& Mou, Xiaomin. 2009. Mandarin Adaptations of Coda Nasals in English Loanwords. Calabrese \& Wetzels, 131-154.

Hsieh, Feng-fan. 2010. Rhyme Phonotactics in Taiwanese: A Dispersion-Theoretic Perspective. Proceedings of the 22nd North American Conference on Chinese Linguistics (NACCL-22) and the 18th International Conference on Chinese Linguistics (IACL-18). Cambridge, MA: Harvard University (pp. 316-30).

Kenstowicz, Micheal. 2012. Cantonese Loanwords: Conflicting Faithfulness in VC Rime Constraints. Catalan Journal of Linguistics 11:65-96.

Keyser, Jay Samuel and Kenneth N. Stevens. 2006. Enhancement and Overlap in the Speech Chain. Language 82:33-63.

Lin, Yen-Hwei. 1989. Autosegmental Treatment of Segmental Processes in Chinese Phonology. Austin, TX: The University of Texas dissertation.

Lindblom, Bjorn. 1963. Spectrographic Study of Vowel Reduction. Journal of Acoustical Society of America 35: 17731781.

Modarresi, Golnaz., Sussman, H., Lindblom, B., \& Burlingame, E. 2004. Stop Place Coding: An Acoustic Study of CV, VC\#, and C\# V Sequences. Phonetica, 61(1): 2-21.

Mou, Xiaomin. 2006. Nasal codas in Standard Chinese: a study in the framework of the distinctive feature theory. Doctoral dissertation, Massachusetts Institute of Technology.

Thomas, Erik R. and Kendall, Tyler. 2007. NORM: The vowel normalization and plotting suite. [Software package]. http://ncslaap.lib.ncsu.edu/tools/norm/

Yu, Alan C. L. 2017. Phonotactic Constraints. Encyclopedia of Chinese Language and Linguistics, General Editor Rint 
Sybesma. Volume 3: 415-422

Zee, Eric. 2003. Frequency Analysis of the Vowels in Cantonese from 50 Male and 50 Female Speakers. Proceedings of the 15th International Congress of Phonetic Sciences (pp. 1117-1120). Universitat Autònoma de Barcelona Barcelona. 